\title{
The Simple Technique of Sternal Closure Using An Absorbable Mesh Plate
}

\author{
Hirotaka Watanuki, MD, Yasuhiro Futamura, MD, Masato Tochii, MD, Kayo Sugiyama, MD, \\ Katsuhiko Matsuyama, MD \\ Department of Cardiac Surgery, Aichi Medical University Hospital \\ Yazakokarimata, Nagakute, Aichi, Japan
}

\section{ABSTRACT}

Background: Several authors have investigated various sternal closure materials and technologies for sternal fixation; nonetheless, the optimal technique for primary sternal closure remains unclear. This study aimed to evaluate the sternal stability of a simple technique using a mesh-type plate (Super Fixorb MX 40 ${ }^{\circledR}$; Takiron Co. Ltd., Osaka, Japan), as compared with wire cerclage.

Methods: A total of 70 patients who underwent cardiovascular surgery through median sternotomy between July 2019 and May 2020 were included. Two pieces of mesh-type plates were placed under the sternum in combination with wiring. The technique for sternal closure was randomly applied, which was mainly based on the surgeon's preferences: mesh plate (mesh group: $N=33$ ) or conventional wire cerclage (wire group: $N=37$ ). Sternal displacement was measured using computed tomography at discharge.

Results: Pain scale scores and analgesic use on postoperative day 7 were similar between the two groups. However, the displacement in both the anterior-posterior and lateral directions was significantly smaller in the mesh group.

Conclusions: The use of the mesh plate device for sternal closure is simple, safe, easy, and potentially reliable without anterior-posterior sternal displacement.

\section{INTRODUCTION}

Although minimally invasive cardiac surgery has gained increasing popularity, conventional full median sternotomy remains to be the standard approach in cardiovascular surgery. Stable sternal closure is an important factor for the reduction of the incidence of postoperative complications. The use of stainless-steel wire cerclage still is the most common sternal closure method. However, simple wire cerclage is likely to cut through the bone, particularly in patients with fragile sternums. Additionally, wire loosening, especially in obese patients, results in the failure to provide adequate stabilization

Received November 24, 2021; accepted December 16, 2021.

Correspondence: Katsubiko Matsuyam, MD, Telephone +81-561-62-3311, Fax +81-561-63-6193 (e-mail: spgfs112@yahoo.co.jp). for optimal sternal healing. Several authors have investigated various sternal closure materials and technologies for sternal fixation [Tam 2018; Nenna 2019]. Nonetheless, the optimal technique for primary sternal closure remains unknown. Recently, the mesh-type plate of an absorbable osteosynthesis device (Super Fixorb MX40 ${ }^{\circledR}$; Takiron Co. Ltd., Osaka, Japan), which is made of unsintered hydroxyapatite polyL-lactic acid (u-HA/PLLA), has been made commercially available as a sternal closure material. Few studies have demonstrated the use of this device [Morimoto 2021]. Hence, the present study aimed to evaluate the sternal stability of a simple technique using a mesh plate device, as compared to that with wire cerclage.

\section{MATERIALS AND METHODS}

Patients: A single-center, observational, retrospective study was conducted at Aichi Medical University Hospital. A total of 99 patients, who underwent cardiovascular surgery through median sternotomy between July 2019 and May 2020, were included as study participants. Patients who did not undergo computed tomography (CT) after surgery and those who required prolonged ventilatory support $(>24$ h) after surgery were excluded. Two techniques were randomly applied, which were mainly based on the surgeon's preferences: mesh plate (Super Fixorb MX40 ${ }^{\circledR}$; Takiron Co. Ltd., Osaka, Japan) (mesh group) and conventional wire cerclage (wire group). Pain (as assessed using a numeric rating scale) and analgesic use were assessed on postoperative day (POD) 7. The sternal displacement in the anterior-posterior and lateral directions was evaluated at discharge by CT scan conducted at $2-\mathrm{mm}$ width intervals. Four axial slices were selected by a radiologist for evaluation. (Figure 1) The study was conducted in accordance with the principles embodied in the Declaration of Helsinki and was approved by the institutional review board. The requirement for the acquisition of informed consent from patients was waived owing to the retrospective nature of this study.

Surgical technique using the mesh plate: Six or seven wires were placed through the sternum, whereas two wires were placed through the manubrium. The distance from the sternal free edge to the wire suture under the sternum was measured at the manubrium and sternal body. The sum of the right-sided and left-sided distances became the width of the mesh. The mesh was cut into two pieces of each width. The sharp parts of the mesh were trimmed into rounded 


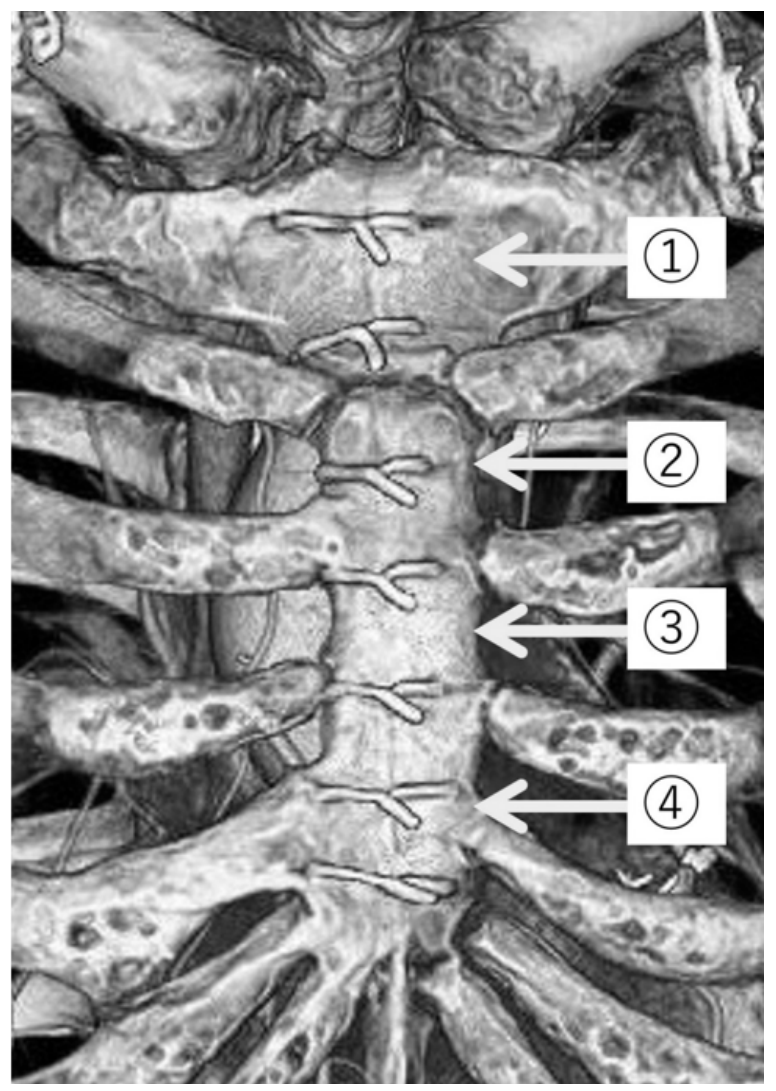

Figure 1. Four axial slices by computed tomography (CT)

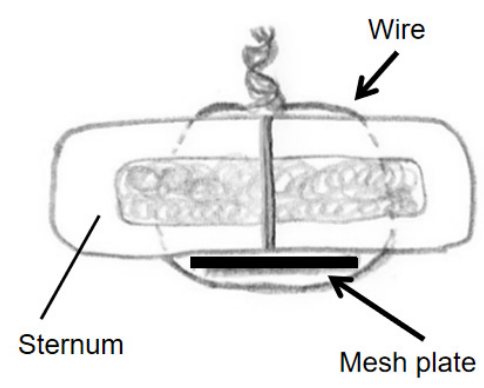

Figure 2. Schematic image of sternal closure using the mesh plate in combination with wiring

corners. After loosely crossing the wire, two pieces of mesh were placed under the sternum using forceps at the manubrium and sternal body. To avoid displacement of the mesh plate from the appropriate position, the wires were tightened. (Figure 2) (Figure 3)

Statistical analysis: Values were expressed as mean \pm standard deviation for continuous variables. Differences in continuous measurements between the two groups were assessed using unpaired Student's t-test. Categorical variables were compared using the chi-squared test or Fisher's exact test. Statistical significance was set at $P<0.05$. All statistical analyses were performed using JMP statistical software version 14.2 (SAS Institute Inc., Cary, NC).

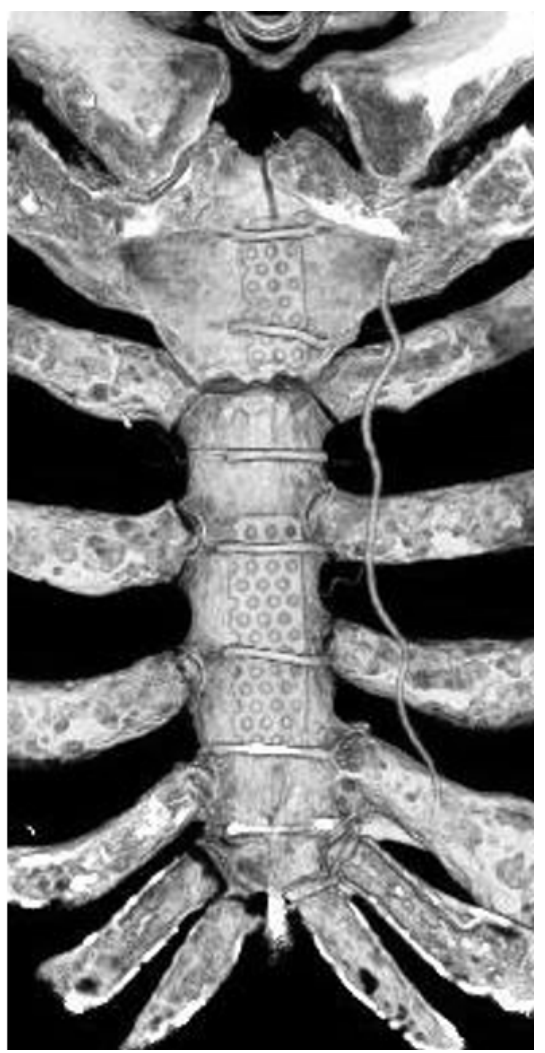

Figure 3. Postoperative CT image after sternal closure with two pieces of mesh plates placed under the sternum

\section{RESULTS}

A total of 70 patients met the criteria for this study. Of these patients, 33 underwent mesh plate fixation (mesh group), whereas 37 underwent conventional wire closure (wire group). The preoperative patient profiles are summarized in Table 1. (Table 1) The mesh group comprised older patients and consisted of fewer individuals with diabetes mellitus. However, multiple regression analysis did not show an independent advantage of the mesh plate.

The operative variables and outcomes are presented in Table 2. (Table 2) There was no statistically significant difference in postoperative chest drainage bleeding between the two groups. No complications associated with the mesh plate were observed. Pain scale scores and analgesic use on POD 7 were similar between the two groups. The superficial sternal wound infection rate was $5.4 \%$ in the wire group and $0 \%$ in the mesh group; nevertheless, no instances of mediastinitis were identified in the two groups. The results of radiographic sternal displacement based on CT findings at discharge are presented in Table 3. (Table 3) Anterior-posterior displacement in all four slices significantly decreased in the mesh group, as compared with the wire group. In contrast, lateral displacement in the sternal body was similar between the two groups. However, sternal displacement in the lateral direction at the manubrium was significantly decreased in the mesh group. 
Table 1. Preoperative patient profiles

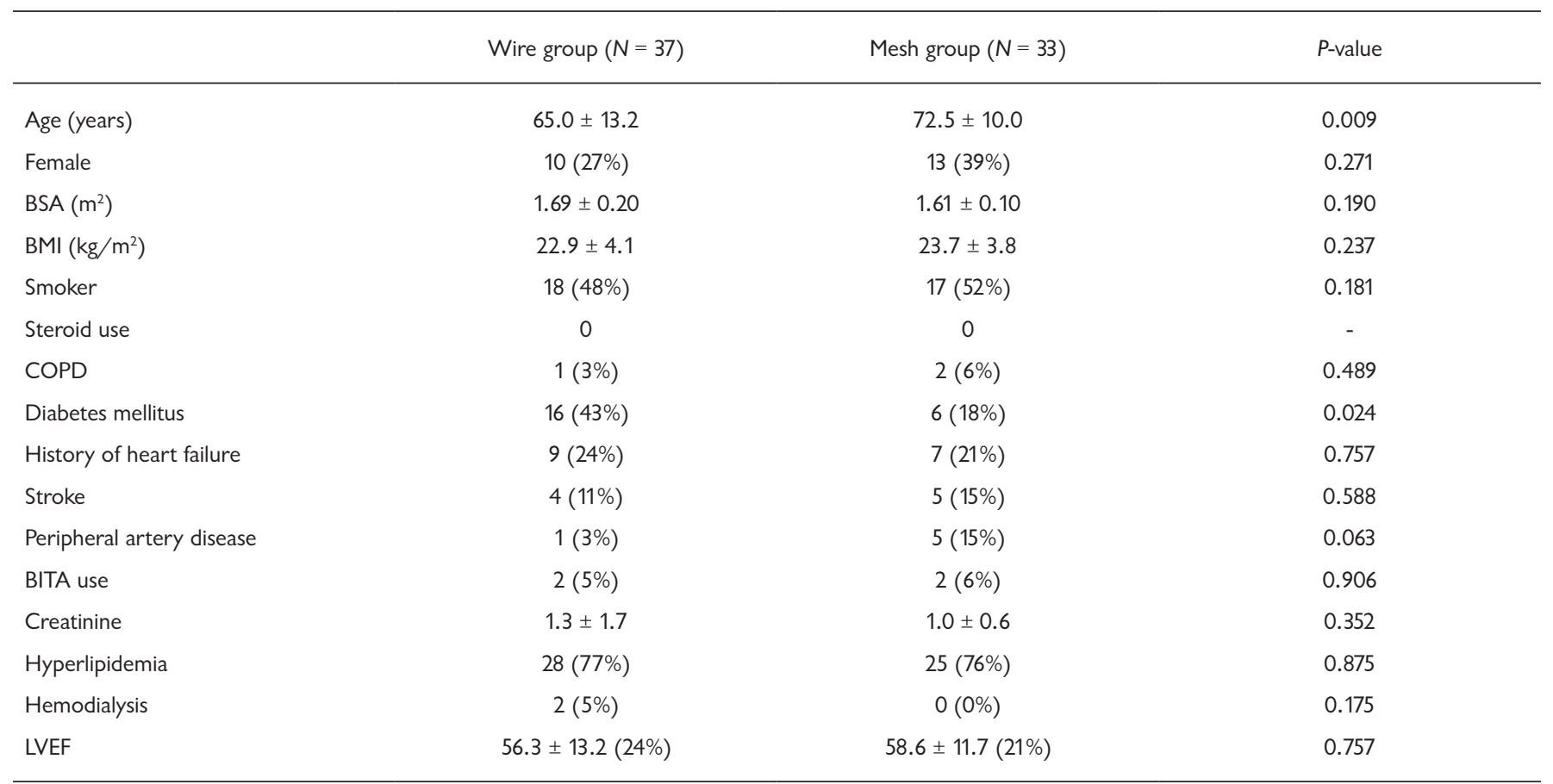

BSA, body surface area; BMI, body mass index; COPD, chronic obstructive pulmonary disease; BITA, bilateral internal thoracic artery; LVEF, left ventricular ejection fraction

\section{DISCUSSION}

Rigid sternal closure can promote early union and primary healing, reduce postoperative pain, improve the upperextremity function and quality of life, and potentially decrease the risk of sternal infection. Although several studies have reported various sternal fixation techniques, little differences in outcomes were detected between various methods and simple closure [Tam 2018]. This may be attributed to numerous factors contributing to sternal wound complications or outcomes.

As an internal fixation device, bioabsorbable osteosynthesis sternal pins (Super Fixorb MX30 ${ }^{\circledR}$; Takiron Co. Ltd., Osaka, Japan) are reportedly able to improve sternal closure stability within a small deviation [Saito 2004]. However, the sternal pins cannot provide sufficient fixation to the fragile bone marrow. Recently, a wave-type device made of u-HA/ PLLA was introduced as an alternative internal fixation device [Kuroki 2020]. This type of device may be more effective in preventing sternal displacement than sternal pins. However, the width or length of the bone marrow space varies depending on the case. Insertion of the wave-type device that matches with the form of the bone marrow may be sometimes difficult. In the case of a rigid bone marrow, there is no sufficient space for the insertion of the wave-type device.

Over recent years, the use of titanium plates and screws has been reported to prevent sternal dehiscence and to result in sufficiently improved sternal healing [Matsuyama 2016]. Some studies showed that rigid plate fixation is superior to wire closure [Nenna 2019; Allen 2017]. However, other studies were unsuccessful in demonstrating this difference [Alhalawani 2013; Tugulan 2020; Snyder 2009]. The plate cannot be firmly affixed to the sternum using a screw in patients with fragile cortical bones. Moreover, as the rigid plate is placed at the anterior cortex of the sternum after dissection of the peristernal fascia, dead space may develop around the plate. The rigid plate does not have bioresorbability and is associated with a potential risk of sternal infection [Wilkinson 1988]. The cost associated with the technique or emergency reopening of the sternum also is a major concern [Allen 2017].

Recently, the rigid mesh-type plate made of u-HA/PLLA (Super Fixorb MX40 ${ }^{\oplus}$; Takiron Co. Ltd., Osaka, Japan) was introduced as a modified sternal closure material in combination with wiring. This mesh-type device is strong enough to prevent sternal cutting by wiring but can be cut into pieces using scissors. Morimoto et al. demonstrated a technique using a mesh-type plate placed both on the anterior wall of the sternum and in the sternal bone marrow in combination with heavy braided polydioxanone [Morimoto 2021]. Although this technique can firmly prevent anterior-posterior sternal displacement, there are times wherein the mesh fails to fit the anterior sternum, due to the presence of muscle or fascia. Mesh plate placement on the sternum also is associated with a potential risk of sternal infection, similar to titanium plate placement.

In this study, we placed the mesh plate under the sternum in combination with wires, which resulted in a good fit. The posterior surface of the sternum had no rugged tissues, such 
Table 2. Operative variables and outcomes

\begin{tabular}{lccl}
\hline & Wire group $(N=37)$ & Mesh group $(N=33)$ & $P$-value \\
\hline Emergent operation & $13(35 \%)$ & $12(36 \%)$ & 0.810 \\
Surgery & & & 0.744 \\
$\quad$ CABG (isolated) & $16(43 \%)$ & $13(39 \%)$ & 0.095 \\
$\quad$ Valvular ( \pm CABG) & $3(8 \%)$ & $0(0 \%)$ & 0.316 \\
Aortic ( \pm CABG) & $18(49 \%)$ & $20(61 \%)$ & 0.479 \\
Cardiopulmonary time (min) & $202 \pm 67$ & $406 \pm 87$ & 0.106 \\
Operation time (min) & $370 \pm 93$ & $173 \pm 95$ & 0.548 \\
Chest-drainage bleeding & & $215 \pm 105$ & 0.541 \\
12h & $161 \pm 86$ & $17.5 \pm 6.5$ & 0.650 \\
24h & $205 \pm 102$ & $0.6 \pm 1.6$ & 0.701 \\
Hospital stay (day) & $18.8 \pm 9.0$ & $10(30 \%)$ & 0.762 \\
Pain scale at POD 7 & $0.4 \pm 0.8$ & $0(0 \%)$ & 0.175 \\
Analgesic use at POD 7 & $10(27 \%)$ & $2(5.4 \%)$ & \\
Superficial SSI & & & \\
\hline
\end{tabular}

CABG, coronary artery bypass grafting; POD, postoperative day; SSI, surgical site infection

Table 3. The sternal displacement based on CT findings

\begin{tabular}{lccc}
\hline Slice & Wire group & Mesh group & $P$-value \\
\hline \multicolumn{2}{l}{ Anterior-posterior displacement $(\mathrm{mm})$} & \\
(1) & $0.93 \pm 0.9$ & $0.26 \pm 0.4$ & $<0.001$ \\
$(2)$ & $0.96 \pm 1.1$ & $0.32 \pm 0.5$ & $<0.001$ \\
(3) & $1.18 \pm 0.8$ & $0.49 \pm 0.6$ & $<0.001$ \\
(4) & $1.07 \pm 0.9$ & $0.51 \pm 0.7$ & $<0.001$ \\
Lateral displacement $(\mathrm{mm})$ & & \\
(1) & $0.53 \pm 0.9$ & $0.18 \pm 0.6$ & 0.003 \\
(2) & $0.26 \pm 0.5$ & $0.20 \pm 0.5$ & 0.418 \\
(3) & $0.63 \pm 0.6$ & $0.50 \pm 0.8$ & 0.277 \\
(4) & $0.52 \pm 0.7$ & $0.33 \pm 0.8$ & 0.079
\end{tabular}

as fat or muscle, and was smoothly flat. Notably, the mesh placement under the sternum was associated with a lower risk of sternal infection. We performed re-sternotomy in a patient using this technique three months after the initial surgery. The mesh plate under the sternum was rigidly attached or united with the sternum, and there were no problems with re-sternotomy.

The greatest advantage of using the mesh plate was simply the prevention of sternal cutting by wiring, although the difference in sternal displacement amounted to $<1 \mathrm{~mm}$ on average at discharge. The mesh plate might be potentially effective even without sternal cutting until the completion of sternal healing, particularly in patients with fragile sternums, obesity, and chronic obstructive pulmonary disease. In this study, sternal separation in the lateral direction at the manubrium was significantly decreased in the mesh group because the manubrium is harder than the sternal body and is less likely to deform. The presence of the mesh plate is supposed to contribute to sternal approximation without sternal cutting even at the hard manubrium.

Postoperative sternal dehiscence can occur within the first two weeks [Wilkinson 1988], and sternal healing usually is complete within 8 to 12 weeks. Unfortunately, this study showed the effectiveness of sternal displacement only during the early postoperative stage and did not evaluate improved sternal healing or long-term results. Moreover, a comparison between this method and other simple alternative methods, such as the figure-of-eight method, might be better.

\section{CONCLUSION}

The use of the mesh plate device for sternal closure is simple, safe, easy, and potentially reliable without anteriorposterior sternal displacement. Further research based on high-quality evidence in the form of a large randomized controlled trial is required to assess the potential benefits of sternal closure using mesh plates.

\section{REFERENCES}

Alhalawani AM, Towler MR. 2013. A review of sternal closure techniques. J Biomater Appl. 28:483-97.

Allen KB, Thourani VH, Naka Y, et al. 2017. Randomized, multicenter 
trial comparing sternotomy closure with rigid plate fixation to wire cerclage. J Thorac Cardiovasc Surg. 153:888-96.

Kuroki H, Arai H, Srisont S, et al. 2020. Corrugated Sheet of Unsintered Hydroxyapatite Poly-L-Lactide for Sternal Fixation: A Preclinical Study. Ann Thorac Cardiovasc Surg. 26:40-6.

Matsuyama K, Kuinose M, Koizumi N, Iwasaki T, et al. 2016. Sternal closure by rigid plate fixation in off-pump coronary artery bypass grafting: a comparative study. J Artif Organs. 19:175-8.

Morimoto K, Matsushita T, Masuda S, et al. 2021. The Novel Technique of Sternal

Closure With Absorbable Mesh for Osteoporotic Patients. Heart Lung Circ. 30:e65-7.

Nenna A, Nappi F, Dougal J, et al. 2019. Sternal wound closure in the current era: the need of a tailored approach. Gen Thorac Cardiovasc Surg. 67:907-16.
Saito T, Iguchi A, Sakurai M, et al. 2004. Biomechanical study of a polyL-lactide (PLLA) sternal pin in sternal closure after cardiothoracic surgery. Ann Thorac Surg. 77:684-7.

Snyder CW, Graham LA, Byers RE, et al. 2009. Primary sternal plating to prevent sternal wound complications after cardiac surgery: early experience and patterns of failure. Interact Cardiovasc Thorac Surg. 9:763-6.

Tam DY, Nedadur R, Yu M, Yanagawa B, et al. 2018. Rigid Plate Fixation Versus Wire Cerclage for Sternotomy After Cardiac Surgery: A MetaAnalysis. Ann Thorac Surg. 106:298-304.

Tugulan CI, Spindel SM, Bansal AD, et al. 2020. Does Elective Sternal Plating in Morbidly Obese Patients Reduce Sternal Complication Rates? Ann Thorac Surg. 110:1898-903.

Wilkinson GA, Clarke DB. 1988. Median sternotomy dehiscence: a modified wire suture closure technique. Eur J Cardiothorac Surg. 2:287-90. 